\title{
A method for isolating RNA from canine bone
}

Rebecca Nance ${ }^{1,2}$, Payal Agarwal1,2, Maninder Sandey², Dmytro Starenki ${ }^{3}$, Jey Koehler ${ }^{2}$, Abdul Mohin Sajib² \& Bruce F Smith ${ }^{\star 1,2}$

${ }^{1}$ Scott-Ritchey Research Center, College of Veterinary Medicine, Auburn University, Auburn, AL 36849, USA; ${ }^{2}$ Department of Pathobiology, College of Veterinary Medicine, Auburn University, Auburn, AL 36849, USA; ${ }^{3}$ HudsonAlpha Institute for Biotechnology, Huntsville, AL 35806, USA; *Author for correspondence: smithbf@auburn.edu

BioTechniques 68: 311-317 (June 2020) 10.2144/btn-2019-0153

First draft submitted: 12 November 2019; Accepted for publication: 11 February 2020; Published online: 14 April 2020

\section{ABSTRACT}

Extracting sufficient quantity and quality RNA from bone is essential for downstream application, such as transcriptomic sequencing, to evaluate gene expression. Isolation of RNA from bone presents a unique challenge owing to the hypocellular, brittle and mineralized matrix, which makes homogenizing the tissue difficult and provides little RNA to work with. Removal of contaminating tissue, such as bone marrow and connective tissue, is essential for isolating RNA that is unique to osteoblasts, osteoclasts and osteocytes. This study established a method to effectively isolate RNA from normal canine bone cells using the phalanges, without contamination from other tissue types, for downstream transcriptomic analysis.

\section{METHOD SUMMARY}

A combination of physical manipulation to remove exterior tissue, washing and centrifugation to remove cells and fat within the diaphysis, homogenization using a mortar and pestle on dry ice prior to bead dissociation, followed by acid guanidinium thiocyanate-phenol-chloroform extraction and column purification yielded sufficient quantity and quality RNA.

KEYWORDS:

bone $\bullet$ canine $\bullet$ dog $\bullet$ osteoblasts $\bullet$ osteoclasts $\bullet$ osteocytes $\bullet$ RNA $\bullet$ sequencing

Isolation of high-quality RNA from tissue is necessary to evaluate the molecular basis of gene expression in that tissue. mRNA, as well as a variety of noncoding RNA, provides insight into gene activity and, therefore, cellular processes and pathways that are active in a given tissue. Common approaches to analysis of RNA include quantitative and end point reverse-transcriptase PCR and transcriptomic sequencing. The quality of RNA obtained is a key factor in validating the significance of RNA sequencing and analysis [1]. Although established methods for RNA isolation often provide sufficient yield, especially for soft tissues, they are not applicable to some tissues, such as bone.

The single-step method of isolating total RNA using an acid guanidinium thiocyanate-phenol-chloroform mixture has been a widely used and well-established technique for more than 30 years [2]. This approach results in cell lysis as well as inhibition of RNases and DNases. With the addition of chloroform, phase separation allows for the isolation of RNA, DNA and/or protein. RNA is precipitated using propanol and a high salt solution. This technique is flexible and variations in the protocol have accommodated many diverse tissues. For example, in tissues with high proteoglycan content such as cartilage, undesirable precipitates are often formed during the RNA precipitation step. In a modification described by Lee et al., an additional phase separation and high salt precipitation avoided the formation of unwanted precipitates [3]. Although the traditional single-step 'Chomczynski' method typically provides high-yield, high-quality RNA, it requires meticulous technique to avoid carryover of unwanted material when pipetting the aqueous layer. As a result, column-based methods have been growing in popularity as a quick and easy alternative but are effectively limited in certain tissues with inadequate yield [3]. Higher yield of RNA is often obtained from the Chomczynski method in comparison to column-based methods [3]. The 'TRIspin method' combines both approaches by following the initial steps of the Chomczynski method with a column-based method [4]. This method was originally developed to provide maximal reproducible amounts of high-quality RNA from dense, hypocellular connective tissue of rabbits [4].

Tissue homogenization is an essential step to releasing RNA from cells. Several methods have been described such as bead dissociation, liquid nitrogen grinding, cryogenic mill pulverization and cryosectioning [3,5-7]. Some tissues present unique challenges for RNA isolation and require altered homogenization protocols to obtain sufficient RNA. Bone in particular is difficult to manipulate owing to the rigid nature of the proteoglycan-rich matrix, which makes homogenizing the tissue challenging and the low cell-to-matrix ratio, which provides little RNA to work with [3].

Bone is a dynamic, living tissue, which is constructed and maintained by three types of cells: osteoblasts, osteoclasts and osteocytes. Osteoblasts, which originate from mesenchymal stem cells, are responsible for the creation of new bone and represent 4-6\% of the total cell population. Osteoclasts, which are of macrophage origin, remove old bone matrix, helping to remodel bone in response to external forces. Osteocytes, the most abundant cells in bone, are differentiated osteoblasts that have become embedded in the bone matrix 
and coordinate the bone remodeling activities of osteoblasts and osteoclasts. The extracellular bone matrix consists of inorganic salts, largely phosphate and calcium ions and an organic matrix composed predominantly of collagenous proteins and some noncollagenous proteins [8]. Most of the bone studies have used formalin fixation, decalcification and paraffin embedding, which results in a highly altered RNA profile and loss of RNA integrity [1]. Cryosectioning is widely considered the best source of intact bone RNA [1]. A method of isolating RNA from osteocytes in human femurs has been established by Eisenberger et al. Their technique employs the use of tape-assisted cryosectioning followed by hematoxylin-eosin staining to guide microdissection using an UVa-nitrogen laser. RNA was then isolated from the microdissected osteocytes via the TRIspin method of Reno et al. [4]. Whereas this method is appropriate for characterizing the profile of osteocytes specifically, it requires equipment that is not always readily available and excludes osteoblasts and osteoclasts, which are additional key components of bone composition. Although osteocytes are the most abundant cell type in bone, characterization of all three cell types is important for evaluating the molecular basis of disease, particularly osteosarcoma.

Contaminating tissue, such as bone marrow and periosteum, are problematic for isolating RNA that is unique to osteoblasts, osteoclasts and osteocytes. The downstream transcriptomic analysis of cancerous versus noncancerous tissue requires the isolation of RNA from tissue of the same origin as that of the tumor. With the exception of a single murine study, current methods for isolating RNA from all three bone cell types do not account for the contaminating marrow RNA [5]. This is especially important as hematopoietic precursor cells express many stem cell markers that could be shared with neoplastic cells, especially the small subpopulation of radioand chemo-resistant neoplastic cells with a more stem-like phenotype [9].

Preventing RNA degradation prior to isolation is an important consideration. Snap freezing samples in liquid nitrogen as early as possible after harvesting has been used as a suitable means of reducing RNA loss [3,6].

In this study, a technique to isolate sufficient quality RNA from canine bone cells for downstream transcriptomic sequencing was established. Samples were obtained from dogs undergoing limb amputation for osteosarcoma. The phalanges were selected as the bone of choice owing to their location distal and distant from the tumor as well as their relative ease of accessibility. Structurally, the phalanges are long bones, with an extended, bone marrow-filled diaphysis composed of dense cortical bone and epiphyseal caps of trabecular bone on either end. The periosteum is an external layer of connective tissue surrounding the bone [10]. A protocol to isolate RNA from bone should include adaptations to address these contaminating tissues and isolate only cellular bone matrix RNA. The resulting protocol yielded sufficient quality RNA suitable for downstream applications.

\section{Materials \& methods}

\section{Sample preparation \& bone marrow removal}

Phalanx bone specimens were obtained from seven dogs undergoing limb amputation for osteosarcoma at the Auburn University College of Veterinary Medicine. Considering that amputations were performed for clinical treatment of disease, the procurement of tissues from amputated legs was exempt from Auburn University Institutional Animal Care and Use Committee review. In all cases, the tumor was at least one joint space proximal to the phalanx. Upon amputation of the leg, the second phalanx was dissected free of the surrounding tissue. Typically, two to three phalanges were obtained from each amputated limb. Phalanx sizes varied based on the breed and size of the dog but were approximately $12 \mathrm{~mm}$ in length and $8 \mathrm{~mm}$ in width after trimming. After obtaining the phalanx, the exterior soft tissue and periosteum were removed using a scalpel, scissors and clean laboratory wipes (Kimwipe; Kimberly Clark, TX, USA). After sufficient soft tissue removal, the epiphyses were removed and discarded using large shearing cutters. The diaphysis was placed into a 1.5-ml microcentrifuge tube and centrifuged at $10,000 \times g$ for $10 \mathrm{~min}$ at room temperature to remove the bone marrow, which was collected and stored at $-80^{\circ} \mathrm{C}$. The bone was then transferred to a clean $1.5-\mathrm{ml}$ microcentrifuge tube, covered with $1 \times$ phosphate-buffered saline (PBS) (Corning, NY, USA) and centrifuged at $10,000 \times g$ for $5 \mathrm{~min}$. After transferring the bone to a clean 1.5-ml microcentrifuge tube, this PBS wash was repeated to ensure the least amount of contaminating bone marrow remained within the medullary cavity. The prepared bone was then snap frozen in liquid nitrogen and stored at $-80^{\circ} \mathrm{C}$ for up to 8 months prior to RNA extraction. The total time for bone preparation was approximately $30 \mathrm{~min}$.

\section{Histological staining}

To verify that the bone samples were free from contaminating tissue, histological staining was performed for both processed and unprocessed samples. The processed bones underwent the bone marrow and periosteum removal protocols, including the outer tissue removal, centrifugation and PBS washing. For the unprocessed samples, the epiphyses were simply removed. The specimens were placed in $10 \%$ formalin, decalcified using $7 \%$ hydrochloric acid and embedded in paraffin. They were cut in $5 \mu \mathrm{m}$ transverse sections and stained using hematoxylin and eosin according to established standard methods.

\section{Bone tissue homogenization \& RNA extraction}

A steel mortar and pestle and metal spatula were made RNAse-free by wiping with RNase Away (Thermo Fisher Scientific, MA, USA), wrapping individually in aluminum foil, baking at $350^{\circ} \mathrm{F}$ for $6 \mathrm{~h}$ or overnight and then storing in $-80^{\circ} \mathrm{C}$. Bone samples were removed from storage in $-80^{\circ} \mathrm{C}$, weighed to compare total yield across various sized dogs and subsequently ground into a fine powder using the mortar and pestle on top of a bed of dry ice. The crushed bone powder was divided evenly into two 1.5-ml microcentrifuge tubes, each containing $1 \mathrm{ml}$ of prechilled Tri-Reagent (Molecular Research Center, $\mathrm{OH}, \mathrm{USA}$ ) and approximately $0.5 \mathrm{~g}$ of zirconium oxide beads (Next Advance, 
ceria stabilized zirconium oxide, $0.5 \mathrm{~mm}$ diameter) and kept on ice. The samples then underwent four rounds of a 30 -s spin in a Bullet Blender (Next Advance) at a speed of '5.5' with a 1-min incubation on ice between rounds. After a 10-min incubation at room temperature, $100 \mu$ of bromochloropropane (Molecular Research Center) was added to each microcentrifuge tube, vortexed thoroughly, incubated for $5 \mathrm{~min}$ at room temperature and then centrifuged at $20,000 \times \mathrm{g}$ for $15 \mathrm{~min}$ at $4^{\circ} \mathrm{C}$.

After removal of the aqueous layer, a second extraction was performed by adding $100 \mu$ l of RNase-free water to the tubes containing the organic layer. After thoroughly mixing the contents, they were centrifuged again at $20,000 \times g$ for 15 min at $4{ }^{\circ} \mathrm{C}$ and the aqueous layer was removed and combined with the aqueous layer from the first extraction. Subsequent experiments determined that this additional extraction step was unnecessary and the RNA yield was not largely affected by omitting this step. The genomic DNA column included in the Qiagen RNeasy Kit (Qiagen, Hilden, Germany) was excluded because initial experiments showed a significant reduction in RNA yield when this column was included in the protocol. As a result, we incorporated DNAse to address genomic DNA contamination by treating the aqueous extract with $10 \mu$ DNAse I with 1/10 volume $10 \times$ DNAse I Reaction Buffer (Thermo Fisher Scientific). The reaction was incubated for $10 \mathrm{~min}$ at room temperature. Following the manufacturer's protocol, Dnase was inactivated by adding $10 \mu \mathrm{l}$ of $25 \mathrm{mM}$ EDTA and heated for $10 \mathrm{~min}$ at $65^{\circ} \mathrm{C}$. Following this step, $20 \mu \mathrm{g}$ of RNA grade glycogen (Thermo Fisher Scientific) was added to each tube in an attempt to increase RNA yield in the subsequent steps. All data shown in this paper were obtained using glycogen; however, subsequent experiments have demonstrated that glycogen does not improve RNA yields (data not shown).

An equal volume of $70 \%$ ethanol was added and mixed well by pipetting. The sample was then applied to an RNA column (Rneasy Micro Kit, Qiagen), placed in a 2-ml collection tube and centrifuged for $1 \mathrm{~min}$ at $21,000 \times \mathrm{g}$ at room temperature (all subsequent centrifugations were performed at room temperature). In many cases, this step was repeated to include all of the sample owing to the maximum loading capacity of $700 \mu$ for the columns. After discarding the eluate, $700 \mu$ l of Buffer RW1 was added to each column, incubated for $2 \mathrm{~min}$ at room temperature and centrifuged for $1 \mathrm{~min}$ at $21,000 \times \mathrm{g}$. The flow through was discarded and $500 \mu \mathrm{l}$ of Buffer RPE was added to each column, incubated for $2 \mathrm{~min}$ at room temperature and centrifuged for $1 \mathrm{~min}$ at $21,000 \times \mathrm{g}$. Then, the flow through was discarded and $500 \mu \mathrm{l}$ of $80 \%$ ethanol (prepared using RNase-free water) was applied to each column, incubated for 2 min at room temperature and centrifuged for $3 \mathrm{~min}$ at $21,000 \times \mathrm{g}$. After the flow through was discarded and the collection tube was replaced, the columns were spun at $21,000 \times g$ for 5 min with the lids open. The collection tube was replaced and $17 \mu$ l of RNase-free water (preheated to $65^{\circ} \mathrm{C}$ ) was applied to the center of the column membrane. After a 10-min incubation at room temperature, RNA was obtained via centrifugation for $5 \mathrm{~min}$ at $21,000 \times \mathrm{g}$. Because the column retained a volume of $2 \mu \mathrm{l}$ according to the manufacturer, the total eluted volume was $15 \mu \mathrm{l}$.

\section{RNA quantity \& quality}

The RNA was quantified using a Nanodrop 2000 instrument and assessed for purity using the absorbance values at 260,280 and 230 nm. Pure RNA is considered to have absorbance ratios A260/280 of 2.0 and A260/230 ratios of 2.0-2.2. The samples were then analyzed using the Agilent Bioanalyzer 2100 (Agilent Technologies, CA, USA) and an RNA integrity number (RIN) derived.

\section{Results \& discussion}

\section{Bone samples}

Bone specimens were obtained from the phalanges of seven dogs undergoing limb amputation for osteosarcoma at the Auburn University College of Veterinary Medicine. Immediately after receiving the sample, care was taken to remove all possible external contaminating nonbone tissue, such as periosteum and connective tissue. After cutting off the epiphyses, centrifugation of the diaphysis yielded a pellet of material assumed to be bone marrow and/or fat from the marrow cavity. Washes of the bone with PBS yielded additional visible material in the buffer.

\section{Histological staining}

Histological staining of processed and unprocessed bone indicated that the diaphysis was free from contaminating tissue. Processed samples were subjected to the contaminating tissue removal protocol, including the mechanical removal of exterior soft tissue and the centrifugation and PBS washing to remove contaminants within the diaphysis. The epiphyses were simply removed from the unprocessed samples, with no further manipulations to the bone. In the unprocessed sections, yellow bone marrow adipocytes can be seen within the diaphysis and external connective tissue envelopes the bone (Figure 1B \& C). The processed section shows a clear diaphysis, free from bone marrow or fat, as well as an uncontaminated external surface (Figure 1A).

\section{RNA preparation}

A steel mortar and pestle was used to hammer the frozen bone into a fine powder and zirconium oxide beads in combination with a Bullet Blender further aided in homogenizing the tissue. Keeping the samples on dry ice minimized the RNA lost to RNases. Alternating cycles of bead dissociation in the Bullet Blender with incubations on ice prevented sample overheating and, therefore, RNA degradation. Tri-Reagent was used to liberate RNA from the bone cells and bromochloropropane allowed phase separation of RNA, DNA and protein so that RNA could be isolated. Our initial experiments using the one-step acid guanidinium thiocyanate-phenol-chloroform extraction method yielded minimal RNA that was highly contaminated, presumably with guanidinium isothiocyanate, based on the absorbance ratios at 260/230. Thus, we implemented the 'TRIspin' method developed by Reno and colleagues that employs the use of an RNA 

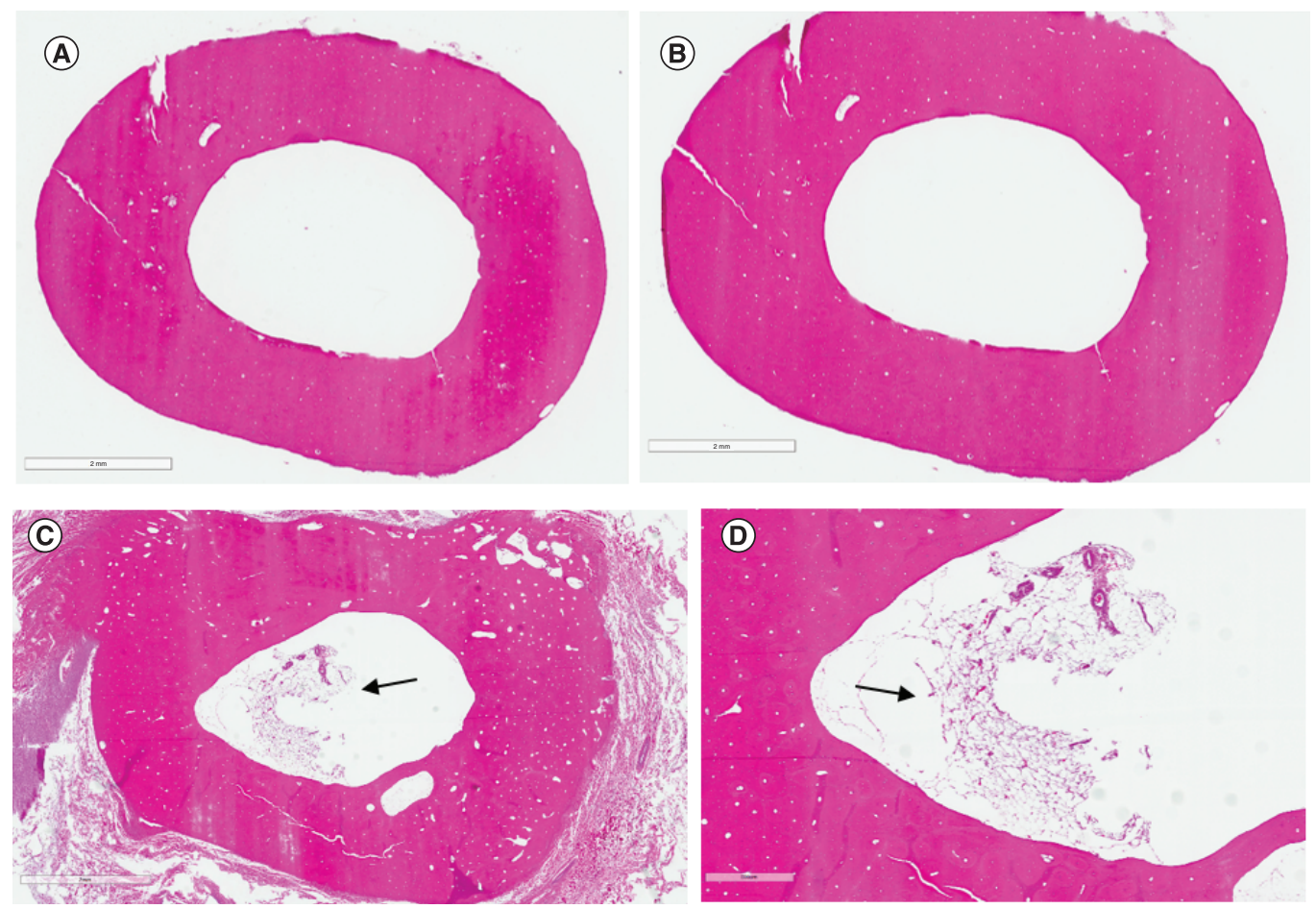

Figure 1. Histological staining of processed versus unprocessed samples to evaluate removal of contaminating nonbone tissue. (A \& B) Hematoxylin and eosin-stained cross-section of phalanx bone after processing to remove contaminating tissue. Processing included physical manipulation to remove exterior connective tissue and the epiphyses, centrifugation to remove tissue within the diaphysis and washes with phosphate buffered saline to further remove any nonbone tissue. The diaphysis and bone exterior appear free from contaminating tissue. (C \& D) Hematoxylin and eosin-stained cross-section of phalanx bone with no manipulations aside from removal of the epiphyses. In addition to connective tissue surrounding the bone, the diaphysis contains what is presumed to be bone marrow or fat (indicated by arrows).

column following the single-step guanidinium isothiocyanate method. This adaptation produced significantly higher yield and quality RNA that proved suitable for downstream transcriptomic sequencing. The genomic DNA column included in Qiagen's RNeasy Column Kit was omitted because of a lack of RNA yield when this column was used. Alternatively, we included a DNase treatment to the extracted RNA prior to applying to the RNA column to eliminate genomic DNA contamination. We attempted to increase RNA yield with a second extraction of the aqueous layer from the organic phenol-chloroform mixture; however, further experiments showed that this did not significantly impact the yield. Glycogen was added in an attempt to further increase RNA yield; however, this did not increase the RNA obtained and in some cases, resulted in a slight decrease in yield (data not shown). RNA was eluted from the column in RNase free water and evaluated for quantity and quality using a Nanodrop instrument and the Agilent Bioanalyzer 2100. In all seven bones, RNA of sufficient quantity and quality, based on Nanodrop data and RIN values, was obtained for transcriptomic sequencing.

\section{Evaluation of RNA quantity \& quality}

Sufficient RNA was obtained in each case to proceed with analysis. RNA concentrations ranged from a low of $3.7 \mu \mathrm{g} / \mathrm{g}$ of tissue to a high of $38.1 \mu \mathrm{g} / \mathrm{g}$ of tissue (Table 1). The mean RNA yield obtained from the bone samples was $14.7 \mu \mathrm{g} / \mathrm{g}$ and the median RNA yield was $12.7 \mu \mathrm{g} / \mathrm{g}$. RNA purity was initially assessed by the ratio of absorbance at $260 \mathrm{~nm}$ to the absorbance at $280 \mathrm{~nm}$. These values ranged from 1.70 to 2.04 , with only one value (1.70) below 2 , indicating high-quality RNA. The ratio of absorbance at $260 \mathrm{~nm}$ to absorbance at $230 \mathrm{~nm}$ ranged from 0.95 to 2.14 , indicating possible carryover of guanidinium isothiocyanate, which absorbs at about $260 \mathrm{~nm}$, in the samples with 260/230 ratios below 2 .

As RNA degradation increases, the $18 \mathrm{~S}$ and $28 \mathrm{~S}$ ribosomal subunit peaks decrease, whereas additional smaller peaks appear. In samples with significant degradation, $18 \mathrm{~S}$ and $28 \mathrm{~S}$ peaks will be difficult to distinguish. In the electrophoretograms pictured in Figure 2 , $18 \mathrm{~S}$ and $28 \mathrm{~S}$ ribosomal subunits are visible as distinct peaks denoted with an asterisk (Figure 2). The initial peak present on the left in each electrophoretogram is the marker used as an internal standard. Although there is variability between the height of rRNA peaks and the presence of additional, sometimes well-defined peaks (as seen in $\operatorname{dog} 7$ ), these electrophoretograms demonstrate moderately intact RNA suitable for sequencing.

RIN is based on an algorithm that evaluates the entire electrophoretic signature to determine a more accurate level of RNA integrity than previous methods that rely on subjective interpretation of electrophoretogram data. RIN is reported as a number ranging from 1 to 10 , with 1 corresponding to highly degraded RNA and 10 equating to mostly intact RNA. RIN values obtained from the seven bone 


\begin{tabular}{|c|c|c|c|c|c|}
\hline Dog & Sample weight (g) & RNA yield $(\mu \mathrm{g})$ & A260/280 & $\mathrm{A} 260 / 230$ & RIN \\
\hline 1 & 0.9778 & 5.5 & 2.01 & 1.52 & 6.90 \\
\hline 2 & 1.1300 & 14.3 & 2.04 & 1.93 & 6.60 \\
\hline 3 & 1.1828 & 16.8 & 2.01 & 1.41 & 6.50 \\
\hline 4 & 0.9788 & 10.2 & 2.03 & 1.82 & 4.50 \\
\hline 5 & 0.5952 & 2.2 & 1.70 & 0.95 & 7.10 \\
\hline 6 & 0.3933 & 15.0 & 2.00 & 2.14 & 7.20 \\
\hline 7 & 1.1372 & 20.8 & $\dagger$ & $\dagger$ & 6.70 \\
\hline
\end{tabular}

$\dagger$ Data not available.

RIN: RNA integrity number.
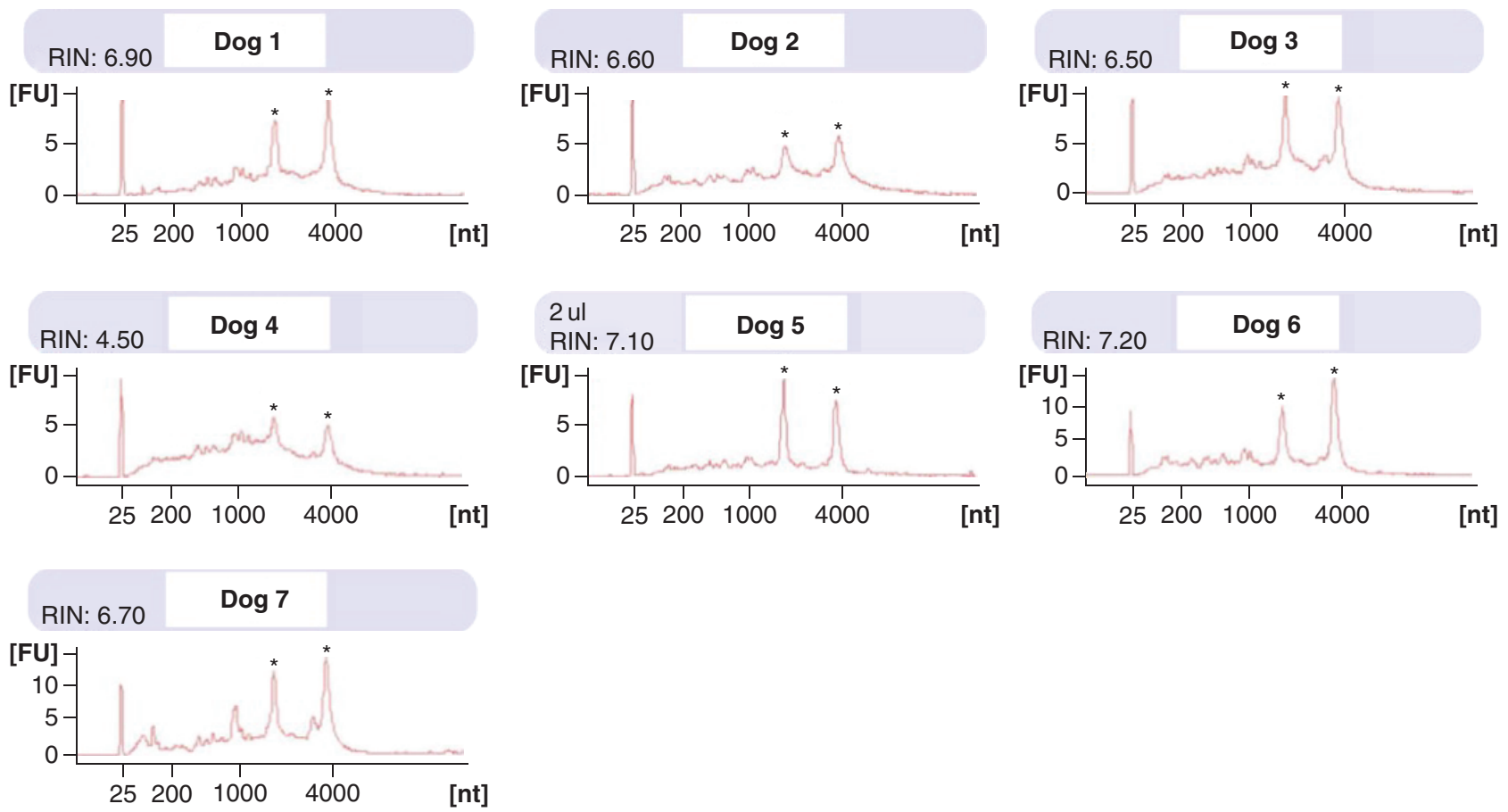

Figure 2. Electrophoretograms of bone RNA. Electrophoretograms showing RNA quality and RIN values obtained for bone RNA using the Agilent Bioanalyzer 2100 . Distinct peaks representing the $18 \mathrm{~S}$ and $28 \mathrm{~S}$ ribosomal subunits are indicated with asterisks (*). RIN values provide a more accurate level of RNA integrity and range from 1 to 10, with 10 indicating fully intact RNA and 1 being completely degraded RNA. The absence of additional significant spikes and anomalies indicates the RNA is of moderate integrity.

FU: Fluorescence unit; RIN: RNA integrity number.

samples ranged from 4.50 to 7.20 , with a mean of 6.54 and a median of 6.70 , indicating moderately intact RNA (Table 1). Dog 4, with the lowest RIN of 4.5, shows the poorest RNA quality, as evidenced by the increased background and decreased rRNA peak distinction present in the electrophoretogram (Figure 2). Nonetheless, this RNA was judged to be of sufficient quality for sequencing.

\section{Conclusion}

Bone composition presents unique challenges to RNA isolation owing to the rigid, hypocellular bone matrix and the presence of contaminating tissue, such as bone marrow, fat and connective tissue. Homogenization of the brittle, mineralized matrix was accomplished using a combination of tissue pulverization using a mortar and pestle on dry ice and bead dissociation, which effectively disrupted the bone matrix prior to RNA liberation using acid guanidinium isothiocyanate-phenol. As expected in a hypocellular tissue such as bone, the total RNA quantity obtained from these samples was relatively low and, therefore, limits the downstream applications. In this case, sufficient amounts for transcriptomic sequencing were achieved. A critical prerequisite for valid transcriptomic sequencing and analysis is high-quality RNA. RIN values confirmed the RNA we obtained was of high integrity.

Removal of unwanted tissue is a critical component of isolating RNA that is unique to bone cells. Although a previous study by Kelly et al. has shown that centrifugation is sufficient to remove bone marrow in the murine femur, a combination of PBS wash and 
centrifugation ensured the least amount of contaminating marrow possible [5]. Although removal of the highly cellular bone marrow results in significantly lower RNA yield owing to the low cell population present in bone, our technique yielded sufficient quantity for sequencing purposes. External soft tissue was removed using physical manipulation. Although histological staining indicated the bone samples were free from external and internal contaminating tissue, it is not possible to conclude that the samples consist solely of RNA from osteoblasts, osteoclasts and osteocytes.

As an alternative to the one-step method of RNA isolation using guanidinium isothiocyanate, which yielded negligible RNA, we adapted the TRIspin method developed by Reno et al. that combines the one-step method followed by an RNA column. This technique provided maximal amounts of high-quality RNA from all seven bone samples.

RNA isolation is a key component of analyzing gene expression and disease characteristics. The difficulties associated with manipulating bone present unique challenges to RNA isolation. Extracting RNA from bone cells independent of contaminating cell types is essential for downstream comparison of gene expression specific to cells of the bone matrix. In this study, we have established a technique for isolating RNA from canine phalanges, independent on contaminating tissue, which successfully yields RNA of sufficient quantity and quality for downstream transcriptomic sequencing.

\section{Future perspective}

Extracting high-quality RNA from bone is critical for evaluating gene expression and disease characteristics. This paper provides a relatively simple method that allows RNA sequencing of canine bone cells. RNA profile analysis of extracted bone cells compared with osteosarcoma has the potential to provide powerful targets for precision medicine. With the advancement of single-cell RNA sequencing, it would be interesting to apply this method to bone cells. Protocols have been established for isolating osteocytes from mouse bone using enzymatic disruption of the tissue matrix using a combination of collagenase and EDTA [11,12]. However, this method has not been published using canine bone, which contains a denser matrix that of mouse. Additionally, our method described herein uses mechanical tissue disruption to obtain RNA and thereby includes the RNA from all bone cells within the matrix, not just osteocytes.

\section{Author contributions}

$\mathrm{R}$ Nance participated in the protocol adaptation design and sample processing [13]. R Nance and BF Smith participated in ongoing research efforts. P Agarwal, J Koehler and BF Smith participated in the overall concept. P Agarwal, AM Sajib and BF Smith participated in the initial protocol design. D Starenki participated in the RNA quality analysis. M Sandey and J Koehler participated in the pathology staining. M Sandey participated in the histological staining.

\section{Acknowledgments}

We are grateful for the assistance of B Matz, A Smith and S Lindley with sample obtainment and overall concept. We are also grateful to $E$ Stickelmaier and the entire oncology service team of the Auburn University College of Veterinary Medicine for providing patient samples.

\section{Financial \& competing interests disclosure}

This study was generously funded by the Scott-Shockley Foundation and the Auburn University Research Initiative in Cancer (AURIC). The authors have no other relevant affiliations or financial involvement with any organization or entity with a financial interest in or financial conflict with the subject matter or materials discussed in the manuscript apart from those disclosed.

No writing assistance was utilized in the production of this manuscript.

\section{Open access}

This work is licensed under the Attribution-NonCommercial-NoDerivatives 4.0 Unported License. To view a copy of this license, visit http://creativecommons.org/licenses/by-nc-nd/4.0/

\section{References}

Papers of special note have been highlighted as: $\bullet$ of interest; $\bullet \bullet$ of considerable interest

1. Eisenberger S, Hoppe G, Pyerin W, Ackermann K. High-quality RNA preparation for transcript profiling of osteocytes from native human bone microdissections. Anal. Biochem. 335(2), 260-266 (2004).

- Provides an alternative method for obtaining specifically osteocytes from bone, which is distinct from our method which includes all bone cells (osteoblasts, osteoclasts and osteocytes).

2. Chomczynski P, Sacchi N. The single-step method of RNA isolation by acid guanidinium thiocyanate-phenol-chloroform extraction: twenty-something years on. Nat. Protoc. 1(2), 581-585 (2006).

3. Lee JTY, Cheung KMC, Leung VYL. Extraction of RNA from tough tissues with high proteoglycan content by cryosection, second phase separation and high salt precipitation. J. Biol. Methods 2(2), 1-3 (2015)

4. Reno C, Marchuk L, Sciore P, Frank CB, Hart DA. Rapid isolation of total RNA from small samples of hypocellular, dense connective tissues. BioTechniques 22(6), 1082-1086 (1997).

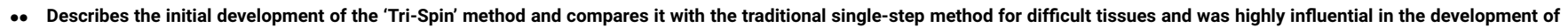
our method.

5. Kelly NH, Schimenti JC, Ross FP, Van Der Meulen MCH. A method for isolating high quality RNA from mouse cortical and cancellous bone. Bone 68, 1-5 (2014).

-. Describes a method of eliminating contaminating bone marrow from the interior of long bones, which we adapted for use.

6. Carter LE, Kilroy G, Gimble JM, Floyd ZE. An improved method for isolation of RNA from bone. BMC Biotechnol. 12(6), (2012). 
7. Cepollaro S, Della Bella E, De Biase D, Visani M, Fini M. Evaluation of RNA from human trabecular bone and identification of stable reference genes. J. Cell. Physiol. 233(6), 4401-4407 (2018)

8. Florencio-Silva R, Sasso GRD, Sasso-Cerri E, Simoes MJ, Cerri PS. Biology of bone tissue: structure, function and factors that influence bone cells. Biomed. Res. Int. doi: 10.1155/2015/421746 (2015) (Epub ahead of print).

9. Kim WT, Ryu CJ. Cancer stem cell surface markers on normal stem cells. BMB Rep. 50(6), 285-298 (2017)

10. Clarke B. Normal bone anatomy and physiology. Clin. J. Am. Soc. Nephro. 3, S131-S139 (2008).

11. Stern AR, Stern MM, Van Dyke ME, Jähn K, Prideaux M, Bonewald LF. Isolation and culture of primary osteocytes from the long bones of skeletally mature and aged mice. BioTechniques 52(6), 361-373 (2012).

12. Shah KM, Stern MM, Stern AR, Pathak JL, Bravenboer N, Bakker AD. Osteocyte isolation and culture methods. Bonekey Rep. 5, 838 (2016).

13. Protocols.io. A method for isolating RNA from canine bone (2020). https://www.protocols.io/view/a-method-for-isolating-rna-from-canine-bone-be86jhze 
